\title{
ESTIMATION OF THE MAXIMUM MULTIPLICITY OF AN EIGENVALUE IN TERMS OF THE VERTEX DEGREES OF THE GRAPH OF A MATRIX *
}

\author{
CHARLES R. JOHNSON $\dagger$ AND CARLOS M. SAIAGO $\ddagger$
}

\begin{abstract}
The maximum multiplicity among eigenvalues of matrices with a given graph cannot generally be expressed in terms of the degrees of the vertices (even when the graph is a tree). Given are best possible lower and upper bounds, and characterization of the cases of equality in these bounds. A by-product is a sequential algorithm to calculate the exact maximum multiplicity by simple counting.
\end{abstract}

Key words. Eigenvalues, multiplicity, symmetric matrix, tree, vertex degrees.

AMS subject classifications. 15A18, 15A57, 05C50, 05C05, 05C07

1. Introduction. Throughout let $T$ be a tree on $n$ vertices, whose degrees are $d_{1} \geq d_{2} \geq \cdots \geq d_{k} \geq 3>d_{k+1} \geq \cdots \geq d_{n}$, and let $\mathcal{S}(T)$ be the set of all real symmetric matrices whose graph is $T$. The diagonal entries of matrices in $\mathcal{S}(T)$ are unconstrained by $T$. For any $n$-by- $n$ matrix $A, m_{A}(\lambda)$ denotes the (algebraic) multiplicity of $\lambda$ as an eigenvalue of $A$, and $m(A)=\max _{\lambda \in \mathbb{C}} m_{A}(\lambda)$, the maximum multiplicity of an eigenvalue of $A$. Then, we let $m(T)=\max _{A \in \mathcal{S}(T)} m(A)$, the maximum multiplicity of an eigenvalue among matrices whose graph is $T$.

It was shown in [2] that $m(T)$ equals the path cover number, $p(T)$, of $T$, the smallest number of vertex disjoint paths of $T$ that cover all the vertices of $T$. It was also shown in [2] that $m(T)=\max [p-q]$ over all ways in which $q$ vertices may be removed from $T$ so as to leave $p$ paths (i.e. the subgraph of $T$ induced by the other $n-q$ vertices consists of $p$ paths, an isolated vertex counting as a path). However, neither of these formulae for $m(T)$, though polynomially computable from $T$, is expressible in terms of overt parameters of $T$, such as the vertex degrees; in fact, there can be no formula for $m(T)$ strictly in terms of the vertex degrees. There are trees with the same degree sequence but different path cover number. For example, the following trees have degree sequence $3,3,2,1,1,1,1$, but, $m\left(T_{1}\right)=3$ and $m\left(T_{2}\right)=2$.

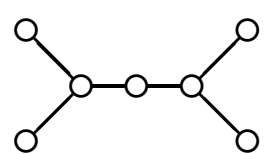

$T_{1}$

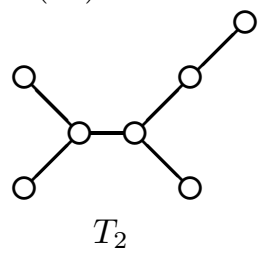

*Received by the editors on 12 December 2000. Accepted for publication on 28 February 2002. Handling Editor: Bryan L. Shader.

${ }^{\dagger}$ Department of Mathematics, College of William and Mary, P.O. Box 8795, Williamsburg, VA 23187-8795, USA (crjohnso@math.wm.edu).

¥Dep. de Matemática, Fac. de Ciências e Tecnologia da Univ. Nova de Lisboa, 2829-516 Quinta da Torre, Portugal (cls@fct.unl.pt). Research supported in part by Fundação para a Ciência e a Tecnologia, Portugal, through the research grant SFRH/BD/899/2000. Part of the research was done while visiting the College of William and Mary. 
Our purpose here is to give simple, tight bounds for $m(T)$ in terms of vertex degrees only, and then to indicate for which trees these bounds are exact. For this purpose, let $H=H(T)$ be the subgraph of $T$ induced by the $k$ vertices of degree at least 3 in $T$. If $H$ consists of a collection of disjoint vertices (possibly empty), we call $T$ segregated; in general $e=e(H)$ is the number of edges present in $H$, so that $e=0$ is equivalent to $T$ being segregated.

2. Main results. Our principal result is Theorem 2.1 whose proof consists of the pendant lemmas. Given a graph $G$ we denote by $\mathcal{V}(G)$ the vertex set of $G$ and by $\operatorname{deg}_{G}(v)$ the degree of a vertex $v$ in $G$.

THEOREM 2.1. Let $T$ be a tree. Then,

$$
1+\sum_{i=1}^{k}\left(d_{i}-2\right)-e \leq m(T) \leq 1+\sum_{i=1}^{k}\left(d_{i}-2\right) .
$$

Equality occurs in the right hand inequality if and only if $T$ is segregated. Equality occurs in the left hand inequality if and only if $\mathcal{V}(H)=\emptyset$ or $\operatorname{deg}_{T}(v) \geq \operatorname{deg}_{H}(v)+2$ for all vertices $v$ of $H$.

As usual, given a graph $G$ with vertex set $\mathcal{V}(G)$ and $U \subset \mathcal{V}(G), G-U$ denotes the subgraph of $G$ induced by the vertex set $\mathcal{V}(G) \backslash U$. Denote by $p_{U}$ the number of components of $G-U$ and by $q_{U}$ the cardinality of $U$. If $U=\{u\}$ and $G$ is a tree, the components of $G-U$ (or $G-u$ ) are called branches of $G$ at $u$.

As we will see, the lower bound presented in Theorem 2.1 is $p_{\mathcal{V}(H)}-q_{\mathcal{V}(H)}$.

LEMMA 2.2. Let $F$ be a forest with c components (trees). Let $F^{\prime}$ be any subtree of $F$ with vertex set $\mathcal{V}\left(F^{\prime}\right)=\left\{u_{1}, \ldots, u_{q}\right\}, q \geq 1$. Then, $F-\mathcal{V}\left(F^{\prime}\right)$ has

$$
c+\sum_{i=1}^{q}\left[\operatorname{deg}_{F}\left(u_{i}\right)-\operatorname{deg}_{F^{\prime}}\left(u_{i}\right)\right]-1
$$

components.

Proof. For each $u_{i} \in \mathcal{V}\left(F^{\prime}\right)$, define $\delta\left(u_{i}\right)=\operatorname{deg}_{F}\left(u_{i}\right)-\operatorname{deg}_{F^{\prime}}\left(u_{i}\right)$, the number of vertices in $F$, but not in $F^{\prime}$, that are adjacent to $u_{i}$. Since $F$ is acyclic and $F^{\prime}$ is a subtree of one component $F^{\prime \prime}$ of $F, \sum_{i=1}^{q} \delta\left(u_{i}\right)$ counts the (distinct) branches of $F^{\prime \prime}$ that are left as components when $\mathcal{V}\left(F^{\prime}\right)$ is removed from $F$. Since $F^{\prime \prime}$ was a component of $F$, there are then $\sum_{i=1}^{q} \delta\left(u_{i}\right)-1$ additional components, $c+\sum_{i=1}^{q} \delta\left(u_{i}\right)-1$ in all, after the removal of $\mathcal{V}\left(F^{\prime}\right)$ from $F$.

Lemma 2.3. Let $F$ be a forest with c components. Consider a nonempty subset $U$ of $\mathcal{V}(F)$ and $F^{\prime}$ the subgraph of $F$ induced by $U$. Assume that $U=\left\{u_{1}, \ldots, u_{q}\right\}$ and $F^{\prime}$ has $e\left(F^{\prime}\right)$ edges. Then, the forest $F-U$ has

$$
p_{U}=c+\sum_{i=1}^{q}\left[\operatorname{deg}_{F}\left(u_{i}\right)-1\right]-e\left(F^{\prime}\right)
$$

components.

Proof. Assume that $F^{\prime}$ has $s$ components (and hence $q-s$ edges), and that $\left\{u_{1}^{\prime}, \ldots, u_{k}^{\prime}\right\}$ are the vertices of a component $F^{\prime \prime}$ of $F^{\prime}$. Then by Lemma 2.2 , the 
removal of $F^{\prime \prime}$ from $F$ will increase the number of components by $\left.\left[\sum_{i=1}^{k}\left[\operatorname{deg}_{F}\left(u_{i}^{\prime}\right)-\operatorname{deg}_{F^{\prime \prime}}\left(u_{i}^{\prime}\right)\right]-1\right]\right]$. Thus the removal of all $s$ components of $F^{\prime}$ from $F$ will increase the number of components to

$$
\begin{aligned}
p_{U} & =c+\sum_{i=1}^{q}\left[\operatorname{deg}_{F}\left(u_{i}\right)-\operatorname{deg}_{F^{\prime}}\left(u_{i}\right)\right]-s \\
& =c+\sum_{i=1}^{q} \operatorname{deg}_{F}\left(u_{i}\right)-\sum_{i=1}^{q} \operatorname{deg}_{F^{\prime}}\left(u_{i}\right)-s \\
& =c+\sum_{i=1}^{q}\left[\operatorname{deg}_{F}\left(u_{i}\right)-1\right]+q-2 e\left(F^{\prime}\right)-s .
\end{aligned}
$$

Since $e\left(F^{\prime}\right)=q-s$ the result follows. that

By Lemma 2.3, we have $p_{\mathcal{V}(H)}=1+\sum_{i=1}^{k}\left(d_{i}-1\right)-e$. Since $q_{\mathcal{V}(H)}=k$ it follows

$$
p_{\mathcal{V}(H)}-q_{\mathcal{V}(H)}=1+\sum_{i=1}^{k}\left(d_{i}-2\right)-e .
$$

Lemma 2.4. Let $F$ be a forest with c components. Let $p$ be the number of components when $q$ vertices each of degree at most 2 are removed from $F$. Then, $p-q \leq c$. Equality occurs if and only if all the vertices removed from $F$ have degree 2 and no two such vertices are adjacent.

Proof. By Lemma 2.3, the removal of $q$ vertices $u_{1}, \ldots, u_{q}$ from $F$ increase the number of components by $\sum_{i=1}^{q}\left[\operatorname{deg}_{F}\left(u_{i}\right)-1\right]-e\left(F^{\prime}\right)$ where $F^{\prime}$ is the subgraph of $F$ induced by $u_{1}, \ldots, u_{q}$. Since all removed vertices from $F$ have degree at most 2 , the number of remaining components is $p \leq c+q$, with equality if and only if all the vertices removed from $F$ have degree 2 and no two such vertices are adjacent.

Given a tree $T$, let $M$ be a subset of $\mathcal{V}(T)$ such that $p_{M}-q_{M}=\max \left[p_{I}-q_{I}\right]$ with $I \subseteq \mathcal{V}(T)$. From Lemma 2.4, there is no vertex of degree 1 in $M$. Lemma 2.4 also implies that we can assume $M$ contains no vertices of degree 2 . In other words, there is a subset $M$ of $\mathcal{V}(H)$ such that $p_{M}-q_{M}=\max \left[p_{I}-q_{I}\right]$ with $I \subseteq \mathcal{V}(T)$ i.e., $p_{M}-q_{M}=m(T)$.

LEMMA 2.5. Let $T$ be a tree. Suppose that $\mathcal{V}(H) \neq \emptyset$ and $\operatorname{deg}_{T}(v)-\operatorname{deg}_{H}(v) \geq 2$ for all vertices $v$ of $H$. Then, $p_{I}-q_{I} \leq p_{\mathcal{V}(H)}-q_{\mathcal{V}(H)}$ for all $I \subseteq \mathcal{V}(H)$.

Proof. Suppose to the contrary, i.e., that there exist $I \subset \mathcal{V}(H)$ such that $p_{I}-q_{I}>$ $p_{\mathcal{V}_{(H)}}-q_{\mathcal{V}(H)}$. Then, there exist $I^{\prime}$ and $I^{\prime \prime}$ such that $I \subseteq I^{\prime} \subset I^{\prime \prime} \subseteq \mathcal{V}(H)$, with $I^{\prime \prime}=I^{\prime} \cup\{v\}$ and $v \notin I^{\prime}$, such that $p_{I^{\prime}}-q_{I^{\prime}}>p_{I^{\prime \prime}}-q_{I^{\prime \prime}}$. Since $q_{I^{\prime}}=q_{I^{\prime \prime}}-1$, $p_{I^{\prime}}-q_{I^{\prime}}>p_{I^{\prime \prime}}-q_{I^{\prime \prime}}$ implies that $p_{I^{\prime}} \geq p_{I^{\prime \prime}}$. But, since $T-I^{\prime}$ has $p_{I^{\prime}}$ components

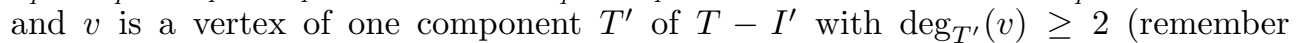
that $\delta(v) \geq 2$ ), by Lemma 2.2 the removal of $v$ from $T^{\prime}$ gives at least 1 additional components. Then, $p_{I^{\prime \prime}} \geq p_{I^{\prime}}+1$ gives a contradiction. $\square$

Lemma 2.6. Let $T$ be a tree. Then, $m(T) \geq 1+\sum_{i=1}^{k}\left(d_{i}-2\right)-e$, with equality if and only if $\mathcal{V}(H)=\emptyset$ or $\operatorname{deg}_{T}(v) \geq \operatorname{deg}_{H}(v)+2$ for all vertices $v$ of $H$. 
Proof. First, observe that if $\mathcal{V}(H)=\emptyset$ i.e., $T$ is a path, then $k=0, e=0$ and $1+\sum_{i=1}^{k}\left(d_{i}-2\right)-e=1$, which is the path cover number when $T$ is a path. Suppose now that $\mathcal{V}(H) \neq \emptyset$ and assume that $\operatorname{deg}_{T}(v) \geq \operatorname{deg}_{H}(v)+2$ for all vertices $v$ of $H$. Consider a vertex set $M$ such that $p_{M}-q_{M}=m(T)$ with $M \subseteq \mathcal{V}(H)$. Since $M \subseteq \mathcal{V}(H)$ and $\operatorname{deg}_{T}(v) \geq \operatorname{deg}_{H}(v)+2$ for all vertices $v$ of $H$ then, from Lemma 2.5, $p_{M}-q_{M} \leq p_{\mathcal{V}(H)}-q_{\mathcal{V}(H)}$. Then, $m(T)=p_{\mathcal{V}(H)}-q_{\mathcal{V}(H)}$ and, by $(2.1)$, $p_{\mathcal{V}(H)}-q_{\mathcal{V}(H)}=1+\sum_{i=1}^{k}\left(d_{i}-2\right)-e$. Therefore, $m(T)=1+\sum_{i=1}^{k}\left(d_{i}-2\right)-e$. Thus, if $\operatorname{deg}_{T}(v) \geq \operatorname{deg}_{H}(v)+2$ for all vertices $v$ of $H$, then equality occurs.

It suffices to prove that if there is a vertex $v$ in $\mathcal{V}(H)$ such that $\delta(v)=\operatorname{deg}_{T}(v)-$ $\operatorname{deg}_{H}(v) \leq 1$, then $m(T)>1+\sum_{i=1}^{k}\left(d_{i}-2\right)-e$. Consider the set $H^{*}=\mathcal{V}(H) \backslash\{v\}$ of vertices of $T$. Then, $T-H^{*}$ has $p_{H^{*}}$ components and $q_{H^{*}}=q_{\mathcal{V}(H)}-1$. Since $v \notin H^{*}, v$ is a vertex of one component $T^{\prime}$ of $T-H^{*}$ and, the removal of $v$ from $T^{\prime}$ gives $\delta(v)-1$ "additional" components. Then, $p_{\mathcal{\nu}(H)}=p_{H^{*}}+\delta(v)-1$ and since $\delta(v) \leq 1, p_{H^{*}} \geq p_{\mathcal{V}(H)}$. Thus, $p_{H^{*}}-q_{H^{*}} \geq p_{\mathcal{V}(H)}-q_{H^{*}}$ and since $q_{H^{*}}=q_{\mathcal{V}(H)}-1$, we have $p_{H^{*}}-q_{H^{*}}>p_{\mathcal{V}(H)}-q_{\mathcal{V}(H)}$. Then, $m(T)>p_{\mathcal{V}(H)}-q_{\mathcal{V}(H)}$ and by (2.1), $p_{\mathcal{V}(H)}-q_{\mathcal{V}(H)}=1+\sum_{i=1}^{k}\left(d_{i}-2\right)-e$. Therefore, $m(T)>1+\sum_{i=1}^{k}\left(d_{i}-2\right)-e$.

LEMMA 2.7. Let $T$ be a tree. Then $m(T) \leq 1+\sum_{i=1}^{k}\left(d_{i}-2\right)$, with equality occurring if and only if $T$ is segregated.

Proof. If $\mathcal{V}(H)=\emptyset$ then $T$ is a path, and hence a segregated tree. In this case, $k=0$ and, $1+\sum_{i=1}^{k}\left(d_{i}-2\right)=1$, which is the path cover number when $T$ is a path. Suppose now that $\mathcal{V}(H) \neq \emptyset$. If $T$ is segregated, for all vertices $v$ of $H, \operatorname{deg}_{H}(v)=0$ and $\operatorname{deg}_{T}(v) \geq 3>\operatorname{deg}_{H}(v)+2$. From Lemma 2.6, since $e=0$ it follows that $m(T)=1+\sum_{i=1}^{k}\left(d_{i}-2\right)$. Thus, if $T$ is segregated, equality occurs.

It suffices to prove that if $T$ is a tree that is not segregated then, $m(T)<1+$ $\sum_{i=1}^{k}\left(d_{i}-2\right)$. Consider a vertex set $M$ such that $p_{M}-q_{M}=m(T)$ and $M \subseteq \mathcal{V}(H)$. From Lemma 2.3, $p_{M}-q_{M}=1+\sum_{v \in M}\left[\operatorname{deg}_{T}(v)-2\right]-e(M)$ where $e(M)$ is the number of edges of the subgraph of $T$ induced by the $q_{M}$ vertices in $M$. If $M=$ $\mathcal{V}(H)$, since $T$ is not segregated, there are, at least, two adjacent vertices of $T$ in $M$ which implies $e(M)>0$. Therefore $p_{M}-q_{M}<1+\sum_{v \in \mathcal{V}(H)}\left[\operatorname{deg}_{T}(v)-2\right]$ i.e., $p_{M}-q_{M}<1+\sum_{i=1}^{k}\left(d_{i}-2\right)$. If $M \subset \mathcal{V}(H), e(M) \geq 0$ and then, $p_{M}-q_{M} \leq$ $1+\sum_{v \in M}\left[\operatorname{deg}_{T}(v)-2\right]$. Since $M \subset \mathcal{V}(H)$ and $\operatorname{deg}_{T}(v) \geq 3$ for all $v \in \mathcal{V}(H)$, $\sum_{v \in M}\left[\operatorname{deg}_{T}(v)-2\right]<\sum_{v \in \mathcal{V}(H)}\left[\operatorname{deg}_{T}(v)-2\right]$, so that $p_{M}-q_{M}<1+\sum_{i=1}^{k}\left(d_{i}-2\right)$.

The proof of Theorem 2.1 is now complete.

3. An algorithm. We close by giving a simple algorithm to compute $m(T)$ by computing the path cover number of $T$.

LEMMA 3.1. Let $T$ be a tree and let $M$ be a subset of $\mathcal{V}(H)$ such that $p_{M}-q_{M}=$ $m(T)$. Then, there is no vertex of degree at least 3 in $T-M$.

Proof. Let $T_{(M)}=T-M$ and suppose that $v \notin M$ and $\operatorname{deg}_{T_{(M)}}(v) \geq 3$. Consider the set $M^{\prime}=M \cup\{v\}$ of vertices of $T$ and the graph $T_{\left(M^{\prime}\right)}=T-M^{\prime}\left(=T_{(M)}-v\right)$. From Lemma 2.2, $T_{\left(M^{\prime}\right)}$ has $p_{M^{\prime}}=p_{M}+\operatorname{deg}_{T_{(M)}}(v)-1$ components and $q_{M^{\prime}}=q_{M}+1$. Since $\operatorname{deg}_{T_{(M)}}(v) \geq 3, p_{M^{\prime}} \geq p_{M}+2$, and $p_{M^{\prime}}-q_{M^{\prime}} \geq p_{M}+2-q_{M^{\prime}}=p_{M}-q_{M}+1$. Thus, $p_{M^{\prime}}-q_{M^{\prime}}>p_{M}-q_{M}$ gives a contradiction. $\square$ 
Lemma 3.2. Let $T$ be a tree and let $M$ be a subset of $\mathcal{V}(H)$ such that $p_{M}-q_{M}=$ $m(T)$. If $v \in \mathcal{V}(H)$ is such that $\delta(v)=\operatorname{deg}_{T}(v)-\operatorname{deg}_{H}(v) \geq 3$, then $v \in M$.

Proof. Let $T_{(M)}=T-M$ and suppose that $v \notin M$ and $\delta(v) \geq 3$. Remember that $M \subseteq \mathcal{V}(H)$ thus, $\operatorname{deg}_{T_{(M)}}(v) \geq \delta(v) \geq 3$. From Lemma 3.1, $\operatorname{deg}_{T_{(M)}}(v) \geq 3$ gives a contradiction. Therefore, $v \in M$.

LEMma 3.3. Let $T$ be a tree and let $M$ be a subset of $\mathcal{v}(H)$ such that $p_{M}-q_{M}=$ $m(T)$. For any $v \in \mathcal{V}(H)$ such that $\delta(v)=\operatorname{deg}_{T}(v)-\operatorname{deg}_{H}(v)=2$, there is a subset $M^{\prime}$ of $\mathcal{V}(H)$ such that $M \subseteq M^{\prime}, v \in M^{\prime}$ and $p_{M^{\prime}}-q_{M^{\prime}}=m(T)$.

Proof. Let $T_{(M)}=T-M$ and suppose that $v \in \mathcal{V}(H)$ with $\delta(v)=2$. If $v \in M$, let $M^{\prime}=M$. Suppose now that $v \notin M$. Recall that $M \subseteq \mathcal{v}(H)$; then $\operatorname{deg}_{T_{(M)}}(v) \geq \delta(v)$. Consider the set $M^{\prime}=M \cup\{v\}$ and graph $T_{\left(M^{\prime}\right)}=T-M^{\prime}\left(=T_{(M)}-v\right)$. From Lemma 2.2, $T_{\left(M^{\prime}\right)}$ has $p_{M^{\prime}}=p_{M}+\operatorname{deg}_{T_{(M)}}(v)-1$ components and $q_{M^{\prime}}=q_{M}+1$. Then, $p_{M^{\prime}}-q_{M^{\prime}}=p_{M}-q_{M}+\operatorname{deg}_{T_{(M)}}(v)-2$.

By Lemma 3.1, $\operatorname{deg}_{T_{(M)}}(v)<3$, hence $\operatorname{deg}_{T_{(M)}}(v)=2$ in which case $p_{M^{\prime}}-q_{M^{\prime}}=$ $p_{M}-q_{M}$. Therefore, $v \in M^{\prime}$ and $p_{M^{\prime}}-q_{M^{\prime}}=m(T)$.

We note that Lemmas 3.2 and 3.3 indicate how to inductively compute a subset $M^{\prime}$ of $\mathcal{V}(H)$ such that $p_{M^{\prime}}-q_{M^{\prime}}=m(T)$. By Lemma 2.4, given any tree $T$, there is a subset $M$ of $\mathcal{v}(H)$ such that $p_{M}-q_{M}=m(T)$ and, by Lemmas 3.2 and 3.3, there is always a subset $M^{\prime}$ of $\mathcal{V}(H)$, containing all vertices $v$ of $T$ with $\delta(v) \geq 2$, such that $M \subseteq M^{\prime}$ and $p_{M^{\prime}}-q_{M^{\prime}}=m(T)$.

Algorithm 3.4. Given a tree $T$, consider the subgraph $H$ of $T$ induced by the vertices of degree at least 3. Remove from $T$ all vertices $v$ of $H$ such that $\delta(v) \geq 2$.

This algorithm is applied to the initial tree $T$ and then to each of the resulting components and so on. Let $M^{\prime}$ be the set of vertices removed via repeated application of Algorithm 3.4. By Lemmas 3.2 and 3.3, all vertices $v$ of $T$ with $\delta(v) \geq 2$ are in $M^{\prime}$. If there is a vertex of degree at least 3 in $T-M^{\prime}$, again by Lemmas 3.2 and 3.3, the application of the Algorithm 3.4 to each of the components of $T-M^{\prime}$ (each one is a tree) allows us to include new vertices in $M^{\prime}$ in route to maximizing $p_{M^{\prime}}-q_{M^{\prime}}$. By Lemma 3.1, the process stops when there is no vertex of degree at least 3 . The set of removed vertices from $T$ gives $M^{\prime}$, the cardinality of $M^{\prime}$ gives $q_{M^{\prime}}$ and the number of components of $T-M^{\prime}$ (each of which is a path) gives $p_{M^{\prime}}$. Finally, the maximum multiplicity of an eigenvalue among matrices whose graph is $T, m(T)$, is equal to $p_{M^{\prime}}-q_{M^{\prime}}$.

\section{REFERENCES}

[1] R. Horn and C. R. Johnson. Matrix Analysis. Cambridge University Press, New York, 1985.

[2] A. Leal-Duarte and C. R. Johnson. The Maximum Multiplicity of an Eigenvalue in a Matrix Whose Graph is a Tree. Linear and Multilinear Algebra, 46:139-144, 1999.

[3] A. Leal-Duarte and C. R. Johnson. On the Minimum Number of Distinct Eigenvalues for a Symmetric Matrix Whose Graph is a Given Tree. Mathematical Inequalities and Applications, To appear. 